\title{
Sum-Rate Maximizing Cell Association via Dual-Connectivity
}

\author{
Minho Kim, Sang Yeob Jung, and Seong-Lyun Kim \\ School of Electrical and Electronic Engineering, Yonsei University \\ 50 Yonsei-Ro, Seodaemun-Gu, Seoul 120-749, Korea \\ Email: \{mhkim, syjung, slkim\}@ ramo.yonsei.ac.kr
}

\begin{abstract}
This paper proposes a dual-connectivity (DC) profile allocation algorithm, in which a central macro base station (MBS) is underlaid with randomly scattered small base stations (SBSs), operating on different carrier frequencies. We introduce two dual-connectivity profiles and the differences among them. We utilize the characteristics of dual-connectivity profiles and their applying scenarios to reduce feasible combination set to consider. Algorithm analysis and numerical results verify that our proposed algorithm achieve the optimal algorithm's performance within $5 \%$ gap with quite low complexity up to $10^{-6}$ times.
\end{abstract}

Index Terms-Dual connectivity, heterogeneous cellular networks, cell association algorithm, capacity maximization.

\section{INTRODUCTION}

Due to spatio-temporal traffic variations coupled with the continuously increasing demand for higher data rates, the heterogeneity is regarded as a key characteristic of the evolving fourth generation $(4 \mathrm{G})$ cellular wireless networks. Deviating from traditional homogeneous macrocellular networks, the $4 \mathrm{G}$ networks are multi-tier heterogeneous cellular networks (HCNs) comprising existing macro base stations (MBSs) overlaid with a diverse set of small base stations (SBSs) such as picocells and femtocells [1]-[3]. Such deployment of SBSs inside the wide coverage area of MBSs enables to provide high capacity and coverage blanket with low power and low cost, resulting in an efficient and cost-effective approach to cater for the expected demand for data [4].

Despite its immense techno-ecocnomic benefits, however, the handover issue should be addressed to support the widescale deployment of SBSs [4], [5]. For example, the higher the density of SBSs, the more handovers between user equipments (UEs) and their serving SBSs will be triggered. This results in (1) extra traffic latency; (2) increased signaling overhead; and

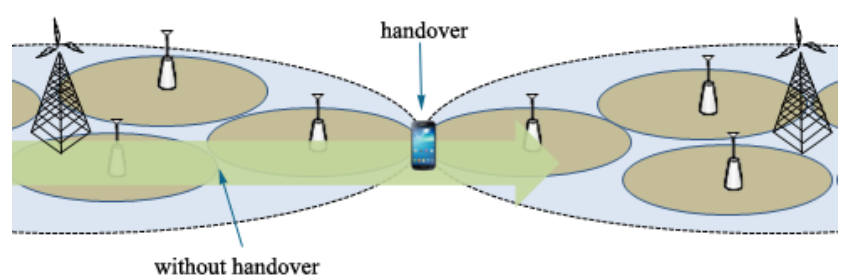

Fig. 1. The concept of Dual-Connectivity.

(3) higher risk in radio link failure (RLF), especially in high

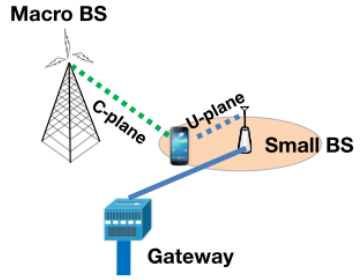

(a) $1 \mathrm{~A}$ profile

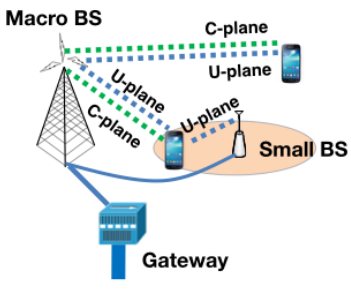

(b) $3 \mathrm{C}$ profile
Fig. 2. Two major types of Dual-Connectivity profiles

mobility scenario [4], [5]. As a remedy, the dual connectivity (DC) stemming from the control plane/user-plane (C/U-plane) split architecture has been actively standardized by the 3rd Generation Partnership Project (3GPP) as part of the Release 12 specifications [see [6]-[8] and the references therein]. As illustrated in Fig. 1, the DC enables a user equipment (UE) to maintain the connection to the MBSs and receive the C-plane data (i.e., signaling messages) from them at all times. The UEs in DC mode needs not to initiate the handover procedure unless moving the coverage of other MBSs, thereby handling the handover issue efficiently.

Even though DC is a promising technology to improve the mobility performance, limited attention has been paid to the capacity maximization, which is another important issue not to be overlooked. There are two major types of DC profiles under consideration by 3GPP: (i) 1-A profile; and (ii) 3-C profile. As depicted in Fig. 2, the UEs receive the U-plane data only from the SBSs in the 1-A profile. In the 3-C profile, on the other hand, the UEs receive the U-plane data from the MBSs and the SBSs simultaneously 11 As one of the solutions for the capacity maximization, the maximum received power-based profile allocation scheme can be exploited, but this may suffer from undesirable scheduling starvation, i.e., the inability to guarantee the quality-of-service for the most UEs. Achieving the capacity maximizing solution while considering the issue of the scheduling starvation is not trivial, due to the multiple number of cases to be allocated, which makes the problem to be NP-hard.

Motivated by the above discussions, in this paper, we

\footnotetext{
${ }^{1}$ Another difference between the 1-A profile and 3-C profile is the traffic flow in a wired network from a Gateway to the MBSs (or SBSs). But we only focus on the wireless traffic flow, throughout the paper.
} 
propose a suboptimal algorithm, which is more efficient but is close to the optimum capacity. The remainder of this paper is organized as follows: Section II describes the system model. Section III formulates the capacity maximization problem. Section IV presents our proposed algorithm. Section V gives numerical results to validate the proposed studies, followed by concluding remarks in Section VI.

\section{SySTEM MODEL}

Consider the downlink of a two-tier HCN consisting of one central MBS serving an area $\mathcal{A}$, overlaid with a set $\mathcal{I}=\{1,2, \cdots, I\}$ of SBSs. We assume that each SBS $i \in \mathcal{I}$ is uniformly distributed in $\mathcal{A}$. Among potential deployment scenarios in DC, we focus on the dedicated-channel deployment scenario in which the MBS and the SBSs operate on different carrier frequencies. Notice that this scenario is prioritized to support the DC in Release 12 in terms of mobility robustness and UE throughput enhancement [5-6]. We define We assume that a set $\mathcal{K}=\{1,2, \cdots, K\}$ of UEs are uniformly and independently distributed in $\mathcal{A}$.

\section{A. Channel Model and UE Association Schemes}

Assume that $P_{m}$ and $P_{s}$ are the transmit powers from the MBS and the SBSs. Over the time-period of interest, all the channel gains are fixed, i.e., the channels remain constant for each time-period, but possibly change over different timeperiods. The channel gain between UE $k$ and SBS $i$ is denoted by $h_{k, i}$, and the channel gain between UE $k$ and the MBS is denoted by $g_{k}$.

Without loss of generality, every UE can be associated with the two-tier HCN simultaneously. In DC, all the UEs receive the C-plane traffic from the MBS for handover robustness. In this context, they should be always associated with the MBS. Assume that UE $k \in \mathcal{K}$ is associated with the MBS. Then, the resulting signal-to-noise ratio (SNR) for UE $k$ can be expressed as

$$
\mathrm{SNR}_{k, m}=\frac{P_{m} g_{k}}{w_{m}},
$$

where $w_{m}=B_{m} n_{m}$ with $n_{m}$ being the power spectral density of the background noise for the MBS.

For the association of SBSs, each UE $k \in \mathcal{K}$ is associated with SBS $i \in \mathcal{I}$ that provides the maximum received power, i.e., $i=\arg \max \left(P_{s} h_{k, i}: k \in \mathcal{K}, i \in \mathcal{I}\right)$. Then, the corresponding single-to-interference-plus-noise ratio (SINR) for UE $k$ is modeled as

$$
\operatorname{SINR}_{k, i}=\frac{P_{s} h_{k, i}}{\sum_{j \in \mathcal{I} \backslash k} P_{s} h_{k, j}+w_{s}},
$$

where $w_{s}=B_{m} n_{s}$ with $n_{s}$ denoting the power spectral density of the background noise for the SBSs.

In practice, the UEs transmit channel quality information or channel status information to the MBS, the MBS can estimate the SINR (or SNR) of every UE [9-12]. Thus, we consider that the MBS can assemble the SINRs (or SNRs) of the UEs in the two-tier $\mathrm{HCN}$, throughput the paper.

\section{B. Loading Model and Scheduling}

For each UE $k \in \mathcal{K}$, the MBS needs to determine one of the DC profiles for capacity maximization. In 1-A profile, UE $k$ receives the U-plane traffic only from the SBSs. In 3-C profile, on the other hand, UE $k$ can receive the U-plane traffic from both the MBS and the SBSs. To be more specific, the MBS can forward some of the U-plane traffic to the SBSs to relay it for UE $k$.

To this end, we assume that the MBS has a Data-FlowEntity (DFE) for each UE $k$. Mathematically, this can be expressed as

$$
D_{k}=\left(D_{k, m}, D_{k, i}\right), \forall k \in \mathcal{K}, \forall i \in \mathcal{I}
$$

where $D_{k, m}, D_{k, i} \in\{0,1\}$, and $m$ and $i$ denote the MBS and SBS $i$ that provides the maximum received power to UE $k$, respectively. Note that $D_{k}=(0,1)$ represents the 1-A profile is allocated to UE $k$, while $D_{k}=(1,0)$ or $D_{k}=(1,1)$ for the $3-\mathrm{C}$ profile.

Under the above framework, we consider a round-robin scheduling mechanism for resource allocation. In this case, we assume that all the UEs are always in service by at least one of the available tiers. For the purpose of exposition, we define the set of UEs receiving the U-plane traffic from the MBS by $\mathcal{K}_{m}=\left\{k \mid D_{k, m}=1\right\} \subset \mathcal{K}$, and the set of UEs receiving the U-plane traffic SBS $i$ by $\mathcal{K}_{i}=\left\{k \mid D_{k, i}=1\right\} \subset \mathcal{K}$. Then, the rate of UE $k \in \mathcal{K}_{m}$ can be written as

$$
R_{k, m}=\frac{B_{m}}{\sum_{k \in \mathcal{K}_{m}} D_{k, m}} \log \left(1+S N R_{k, m}\right),
$$

where the MBS allocates the frequency bandwidth $B_{m}$ evenly to the UE $k \in \mathcal{K}_{m}$. Similarly, the rate of UE $k \in \mathcal{K}_{i}$ is

$$
R_{k, i}=\frac{B_{s}}{\sum_{k \in \mathcal{K}_{i}} D_{k, i}} \log \left(1+S I N R_{k, i}\right) .
$$

\section{PROBLEM Formulation}

In this section, we now consider the DC profile allocation (DCPA) problem over the two-tier HCN. To this end, the optimization problem for capacity maximization can be formulated as follows:

$$
\begin{aligned}
\text { DCPA : } \max _{D_{k, m}, D_{k, i}} & \sum_{k \in \mathcal{K}_{m}} R_{k, m}+\sum_{i \in \mathcal{I}} \sum_{k \in \mathcal{K}_{i}} R_{k, i}, \\
\text { s.t. } & D_{k, m} \in\{0,1\}, \forall k \in \mathcal{K}, \\
& D_{k, i} \in\{0,1\}, \forall k \in \mathcal{K}, \forall i \in \mathcal{I},
\end{aligned}
$$

where $\mathcal{K}_{m}=\left\{k \mid D_{k, m}=1\right\}$, and $\mathcal{K}_{i}=\left\{k \mid D_{k, i}=1\right\}$.

Note that this problem is not straightforward to solve, since it is the integer programming problem (i.e., all of the decision variables take on the value 0 or 1 ). This indicates that the problem is intractable and thus heuristic methods should be exploited instead. In the following section, we will study how to design a sub-optimal algorithm. 


\section{Proposed Algorithm}

One of the optimal solutions for Problem DCPA is the brute-force algorithm that takes into account all possible combinations of data flow, $D_{k}$. It is hard to solve Problem DCPA with the brute-force algorithm since it has an extremely large number of cases to consider. In case of $K$ UEs in the network, the number of possible combinations to check is $3^{K}$ since there three options for each users:

$$
D_{k}=\left(D_{k, m}, D_{k, i}\right)=(1,1) \operatorname{or}(1,0) \operatorname{or}(0,1) .
$$

We devise a sub-optimal algorithm to reduce the complexity of brute-force algorithm. In the first step, the proposed algorithm creates a primitive matrix by $K$ rows by $I+1$ columns, and set a UE identity $k$ at position $(k, i)$ of the primitive matrix if $D_{k, i}$ equals one. If $D_{k, m}$ equals one, set a UE identity $k$ at position $(k, I+1)$ of the matrix. Then convert the primitive matrix to an initial matrix by sorting its columns in descending order.

To simplify the notation, we identify $m$ with $I+1$ in this section. Hence the largest $i$ connote the identity of an MBS e.g. $D_{k, m}=D_{k, I+1}$, and BS $i$ is an MBS for $i=I+1$ or an SBS for $i<I+1$. The initial matrix implies that the DC profile of each UE in a network is $3 \mathrm{C}$ type that both an MBS and one of SBSs serve a UE simultaneously. The proposed algorithm change the individual DC profiles of some UEs in the initial matrix for maximizing network capacity, to either $1 \mathrm{~A}$ or another $3 \mathrm{C}$ at which only an MBS serves downlink transmission to the UE.

Proposition 1 (Necessary condition to be an optimal solution) To maximize the total network capacity in (6), the UE groups that each BS $i$ serves include a UE which have the highest SINR toward the BS $i$.

Proof : Let $G_{o p t, i}$ denote the group of UEs that $B S_{i}$ serves in an optimal solution, $G_{o p t, i}=\left\{U_{1, i}, U_{2, i}, \cdots, U_{n, i}\right\}$ and $\operatorname{SINR}_{k, i}>\operatorname{SINR}_{k+1, i}$ for $k \in \mathcal{K}_{i}$, where $\operatorname{SINR}_{k, i}$ denotes SINR value at $U_{k, i}$ toward BS $i . U_{\bar{k}, i}$ denote the UE which is placed at $(1, i)$ in initial matrix. We show that $U_{1, i}=U_{\bar{k}, i}$ by reductio ad absurdum.

We assume that $U_{1, i} \neq U_{\bar{k}, i}$. Then the UE corresponding to $U_{\bar{k}, i}$ is placed in another column $i^{\prime} \neq i$ because at least one BS serve each UE. There exists another valid combination which is equal to the optimal solution except for $G_{o p t^{\prime}, i}=$ $G_{\text {opt }, i} \cup\left\{U_{\bar{k}, i}\right\}$. Total throughput of BS $i$ in the optimal solution and the alternative solution, can be described as follows respectively:

$$
\text { optimal solution: } \quad \sum_{k \in \mathcal{K}_{i}}\left[R_{k, i}\right]=\sum_{k \in \mathcal{K}_{i}}\left[\frac{B_{i}}{n} \cdot \log \left(1+\operatorname{SINR}_{k, i}\right)\right] \text { (9) }
$$

alternative solution:

$$
\begin{aligned}
& \sum_{k \in \mathcal{K}_{i} \cup\{1\}}\left[R_{k, i}\right] \\
= & \sum_{k \in \mathcal{K}_{i} \cup\{1\}}\left[\frac{B_{i}}{n+1} \cdot \log \left(1+\mathrm{SINR}_{k, i}\right)\right](10)
\end{aligned}
$$

Subtracting (9) from (10) yields

$$
\begin{array}{r}
\frac{B_{i}}{n+1} \log \left(1+\operatorname{SINR}_{\bar{k}, i}\right) \\
-\left[\sum_{k=1}^{n} \frac{B_{i}}{n(n+1)} \log \left(1+\mathrm{SINR}_{k, i}\right)\right]
\end{array}
$$

Substituting $n /(n+1) n$ for $1 /(n+1)$ in 11 yields

$$
=B_{i} \sum_{k=1}^{n}\left[\frac{1}{n(n+1)} \log \left(\frac{1+\operatorname{SINR}_{\bar{k}, i}}{\left.1+\operatorname{SINR}_{k, i}\right)}\right)\right]
$$

(12) is positive because $\operatorname{SINR}_{\bar{k}, i}>\operatorname{SINR}_{k, i}$ for $k \in \mathcal{K}_{i}$. Then, the sum of total throughput of each BS in the alternative solution is greater than the optimal solution's. Thus, $U_{1, i}=$ $U_{\bar{k}, i}$ in an optimal solution.
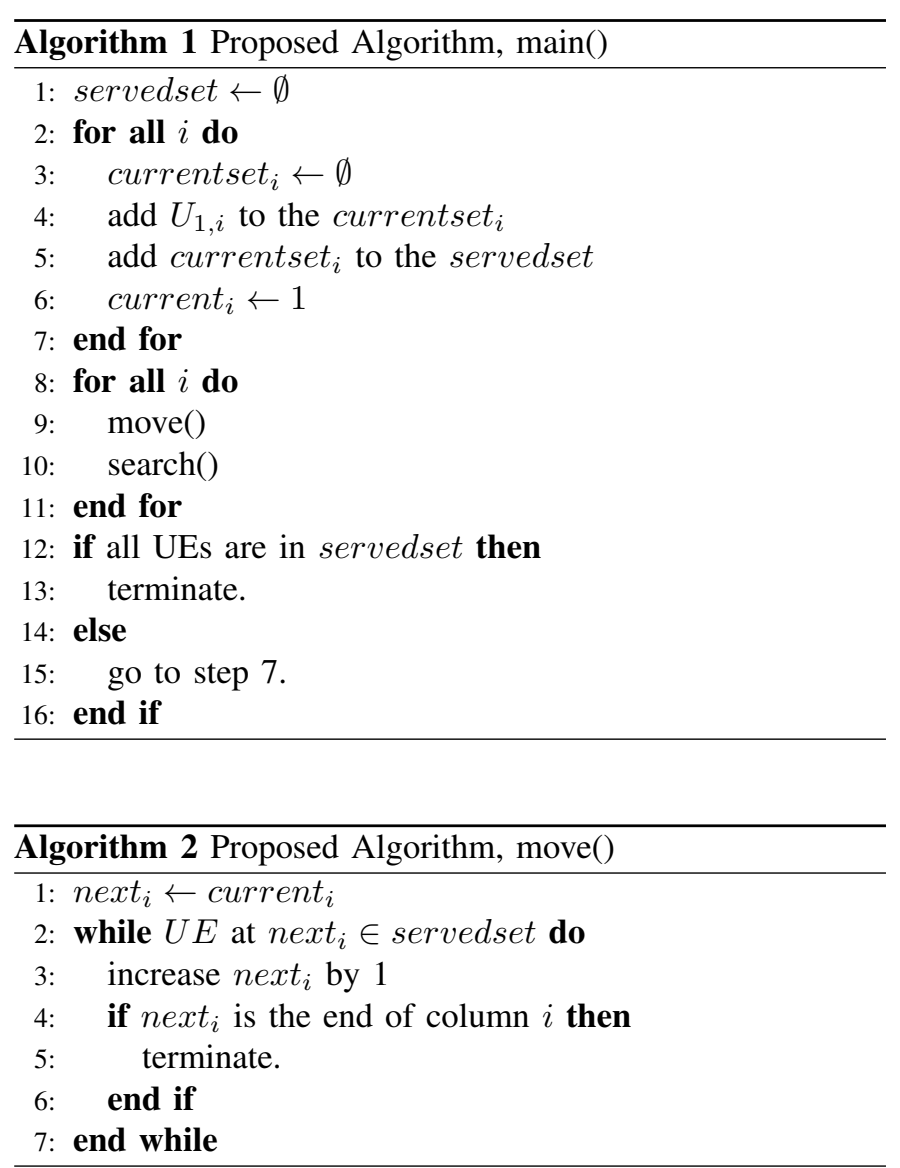

A maximum throughput of the network can be achieved when only top SINR UEs in each BS area are in service. The basic concept of the proposed algorithm is that a suitable UE that minimizes the throughput degradation is admitted, until all the UEs are in service by at least one BS. Using Proposition 1 , the proposed algorithm is summarized above.

\section{Worst-Case Complexity}

The worst case complexity of the proposed algorithm is $2^{K-1}-2^{N-1}+(K-N)(N-1)$, where $K$ and $N$ denote the numbers of UEs and BSs (including both MBSs and SBSs) respectively. The worst case complexity can be 


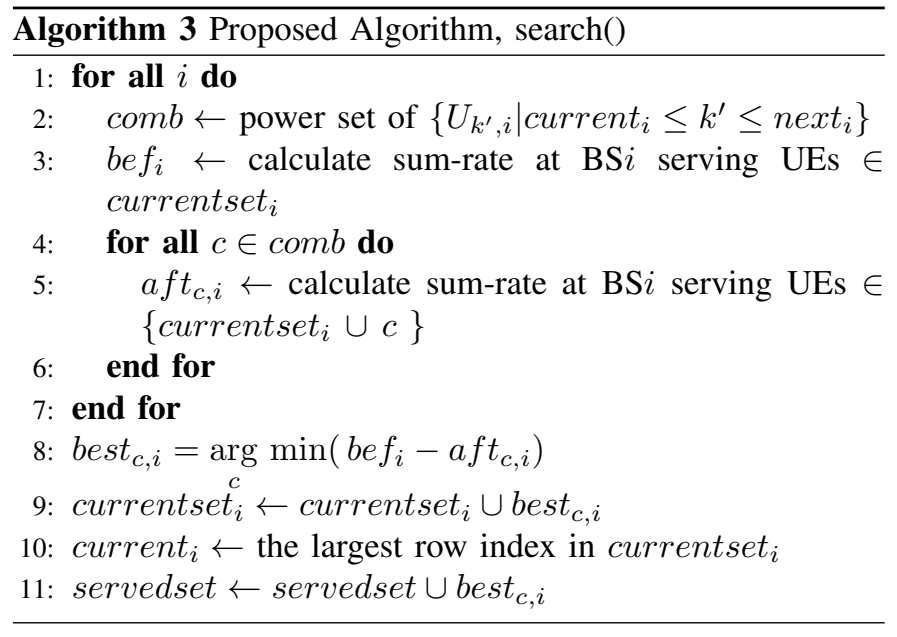

obtained by considering the situation when adding UEs to the servedset which are selected only in SBSs, because it makes a large gap between current $_{\text {macro }}$ and next macro $_{\text {at }}$ search(). The number of all possible combinations at $\operatorname{search}()$ is 2 . (next $_{\text {macro }}-$ current $\left._{\text {macro }}\right)$ and it becomes the dominant term in complexity so that the complexity situated above is formulated as follows,

$$
\begin{aligned}
& \underbrace{\overbrace{2^{N-1}}^{\text {macro }}+\overbrace{N-1}^{\text {small }}}_{1^{\text {st }} \text { iteration }}+\underbrace{2^{N}+N-1}_{2^{\text {nd } \text { iteration }}}+\cdots+\underbrace{2^{K-2}+N-1}_{(K-N)^{\text {th }} \text { iteration }} \\
= & 2^{K-1}-2^{N-1}+(K-N)(N-1)
\end{aligned}
$$

whereas the complexity of an optimal algorithm but bruteforce algorithm is $K \cdot 3^{K}$ since each combination needs $K$ number of rate calculations.

\section{Performance Evaluation}

We show the result of the proposed sub-optimal algorithm's performance by comparing with the full-search optimal algorithm. The result includes comparisons with not only the optimal solution, but also other conventional algorithms such as assigning either $1 \mathrm{~A}$ or $3 \mathrm{C}$ profile to all UEs and assigning a $\mathrm{UE}$ to the only one closest BS. The specific parameters used in our simulation are summarized in Table I Note that the power spectral density $n_{m}$ for an MBS is higher than $n_{s}$. It is to reflect interference coming from other MBSs which could exist if there were multiple MBSs. In ' $3 \mathrm{C}$ only' algorithm, all UEs in a network are in service by both BSs (MBS and $\mathrm{SBS}_{2}^{2}$, whereas in '1A only' algorithm, all UEs are in service by an SBS only. In 'Stronger' algorithm, a UE is in service by more closer BS between the MBS and the SBSs. All results in this section are outcomes under four SBSs with a single MBS. Each result is based on Monte Carlo simulation routines.

We show first, the proposed algorithm's performance by comparison to the optimal solution's capacity as depicted at Fig. 3 It shows that the numerical results of the proposed

\footnotetext{
${ }^{2}$ We only consider $3 \mathrm{C}$ that assign both an MBS and an SBS for downlink transmission even if it is possible to assign an MBS only for downlink transmission.
}

TABLE I

Simulation PARAMETERS

\begin{tabular}{|c||c|}
\hline Network Size & $500 \mathrm{~m} \times 500 \mathrm{~m}$ \\
$\alpha_{\text {macro }}$ & 4.5 \\
$\alpha_{\text {small }}$ & 5 \\
Macro Cell Bandwidth & $10 \mathrm{MHz}$ \\
Small Cell Bandwidth & $10 \mathrm{MHz}$ \\
Macro TX Power & $46 \mathrm{dBm}$ \\
Small TX Power & $20 \mathrm{dBm}$ \\
$n_{m}$ for Macro Cell & $-90 \mathrm{dBm} / \mathrm{Hz}$ \\
$n_{s}$ for Small Cell & $-140 \mathrm{dBm} / \mathrm{Hz}$ \\
\hline
\end{tabular}

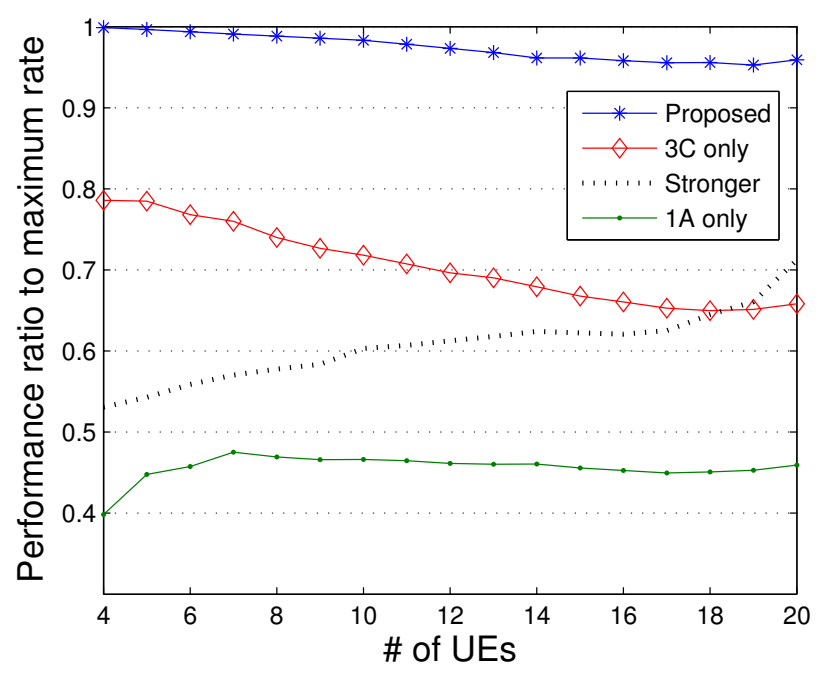

Fig. 3. Performance Ratio of The Proposed algorithm to the Optimal Solution

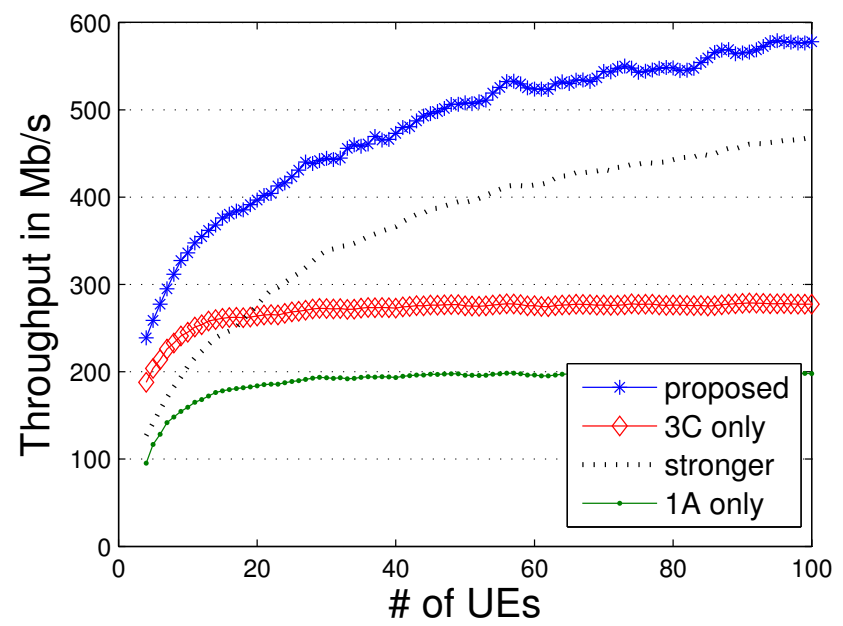

Fig. 4. Performance comparison with other algorithms 


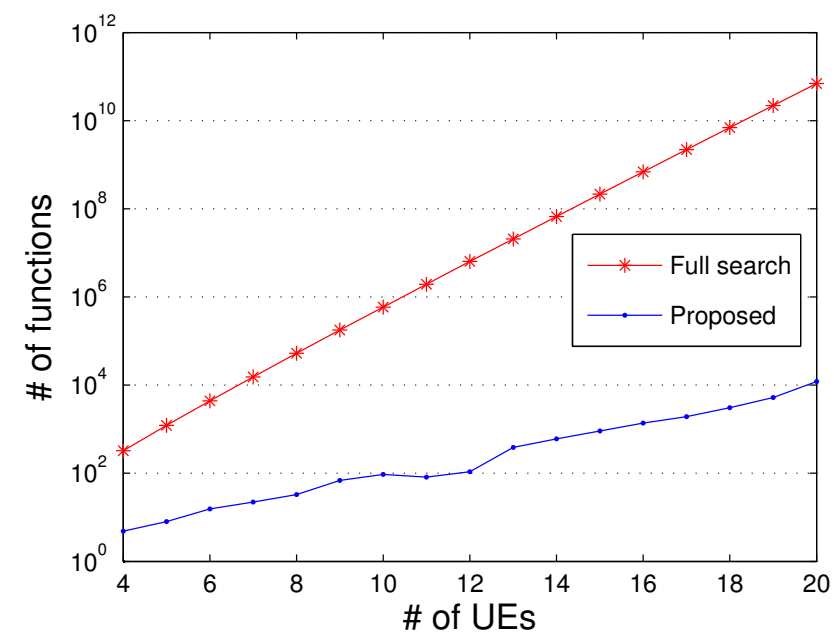

Fig. 5. Complexity comparison with full search algorithms

algorithm are comparable to the optimal solution within $5 \%$ gap. On the other hand, applying single profile algorithm such as '3C only' and ' $1 \mathrm{~A}$ only' do not increase performance as the number of UEs increases because higher UE density do not make UE's SINR better on average. In case of 'Stronger' algorithm, though it increases network capacity, there is still considerable difference to the optimal solution.

Fig. 4 shows the network capacities for each algorithms except the optimal solution, due to the enormous computation cost of it. The proposed algorithm makes the network capacity increase as the number of UEs increase. It comes from that the proposed algorithm provide more frequency resource to better SINR UE by increasing opportunity for allocating both MBS and SBS. Despite the 'Stronger' algorithm also make the network capacity increase, there is around $30 \%$ gap to the proposed algorithm.

The comparison result for the complexity to the full search algorithm is shown in Fig. 5. We analyze the computational complexity of the algorithms by counting the functions of rate calculation in (4) and (5). Although both complexities of each algorithm increase exponentially as the number of UEs increases, the proposed algorithm's complexity does not exceed $10^{4}$ in most cases with the number of UEs not exceeding 20 UEs. Note that the complexity gap between full search and the proposed algorithm increases exponentially so that it is more than $10^{6}$ times for a number of UEs greater than 16. At that time, the proposed algorithm sustains its high performance.

\section{CONCLUSION}

We have investigated the DC profile allocation problem to maximize the network capacity in HCNs. Although an optimal algorithm achieves maximum sum rate capacity, it is hard to apply it because of its exorbitant complexity. In this paper, we introduced a sub-optimal algorithm that makes network capacity close to maximum sum rate for a few UEs (not greater than 20), but considerably lower complexity. The simulation result reveals that the proposed algorithm achieves $97 \%$ of the maximum capacity and low complexity up to $10^{-6}$ times compared with the full search algorithm in terms of the number of executed rate-calculation operations.

\section{REFERENCES}

[1] J. Hoydis, M. Kobayashi, and M. Debbah, "Green small-cell networks," IEEE Veh. Technol. Mag., vol. 6, no. 1, pp. 37-43, Mar. 2011.

[2] I. Hwang, B. Song, and S. Soliman, "A holistic view on hyper-dense heterogeneous and small cell networks," IEEE Commun. Mag., vol. 51, no. 6, pp. 20-27, Jun. 2013.

[3] H. Claussen, "Performance of macro-and co-channel femtocells in a hierarchical cell structure," in Proc. IEEE 18th Intl Symp. on Personal, Indoor and Mobile Radio Commun. (PIMRC07), pp. 1-5, Sep. 2007.

[4] S. Parkvall, E. Dahlman, G. Jongren, S. Landström, and L. Lindbom, "Heterogeneous network deployments in LTE," Ericsson Review, vol. 90, pp. 34-38, 2011.

[5] T. Nakamura and et al., "Trends in small cell enhancements in lte advanced," IEEE Commun. Mag., vol. 51, no. 2, pp. 98-105, Feb. 2013.

[6] 3GPP TR 36.842., "Study on Small Cell Enhancements for E-UTRA and E-UTRAN-Higher layer aspects," 2013.

[7] 3GPP TR 36.932., "Scenarios and Requirements for Small Cell Enhancements," 2013.

[8] 3GPP, "3GPP work items on Small Cells v0.0.4," 2014.

[9] 3GPP TS 36.213., "Evolved Universal Terrestrial Radio Access (EUTRA); Physical layer procedures," 2013.

[10] 3GPP TS 36.300., "Evolved Universal Terrestrial Radio Access (EUTRA) and Evolved Universal Terrestrial Radio Access Network (EUTRAN); Overall Description; Stage 2," 2008.

[11] 3GPP TS 36.314., "Evolved Universal Terrestrial Radio Access (EUTRA); Layer 2 - Measurements," 2011.

[12] 3GPP TS 36.331., "Evolved Universal Terrestrial Radio Access (EUTRA); Radio Resource Control (RRC); Protocol specification,” 2013. 